6. Redfern, M. Grow your own. Information and library manager, 9(4), 1990, pp.4-6.

7. Op. cit. 3

8. Management Charter Initiative. London: Department of Employment, 1995.

9. NHS Training Division and ASSIST Statement of Recognition. Bristol: NHSTD, 1995.

10. Details about the Checkpoint questionnaire for managers can be obtained from: IM\&T Programme Team, NHS Training Division, St. Bartholomews Court, 18 Christmas Street, Bristol, BS1 5BT.

11. Op. cit. 1

12. Tremblay, M. and Wall, A. Chief Executives learning together: a report for the NHS Training Directorate. Birmingham: University of Birmingham Health Services Management Centre, 1994.

The following two authors, Victoria Manglano Bosch and LizMackie were winners respectively, of the 1995 LIRG Postgraduate Student Prize and the Undergraduate Student Prize.

\title{
CD-ROM user interface evaluation: the appropriateness of GUIs
}

VICTORIA MANGLANO BOSCH, Department of Information Science,

City University (London)

\section{Introduction}

The study's general objective was to assess the appropriateness of Graphical User Interfaces (GUI) for CD-ROM applications and examine to what extent a GUI based on Windows could facilitate the ease of use of these applications. A full account of the study is reported elsewhere ${ }^{(1)}$.

The literature review identified three main problematic areas in relation to the acceptance of CDROMs: contradictory perceptions about CD-ROMs, standardization, and the need for evaluation of interfaces.

Contradictory perceptions about CD-ROMs from publishers, users and informatiøn professionals: the need for end user training.

Although publishers define CD-ROMs as 'user-friendly' and particularly suited to end users, and users are reported to be extremely attracted to and satisfied with CD-ROM products, information professionals involved in the management of CD-ROM services realized that end user searching is problematic. Studies investigating the searching behaviour of end-users have indicated the difficulties naive users encounter using CD-ROMs and the inefficiency of their search techniques 
and results. This can be caused not only by the lack of searching skills training but also by the inadequacies of the CD-ROM interfaces. Although CD-ROMs are supposedly easy to use, the amount of attention paid to staff and end user training would suggest otherwise.

\section{The problem of standardization}

Two of the main limitations of the adoption of CD-ROMs is the lack of a standardized retrieval software and the proliferation of interfaces, because both end users and professionals have to learn to use several interfaces from different producers. The need for CD-ROM standards is a common issue in the literature and there are significant efforts within the library community to establish standards. To what extent the adoption of GUIs could be considered a 'de facto' standard, as it has been suggested, was explored in the evaluation.

\section{The need for evaluation of interfaces}

Although several models for the evaluation of $\mathrm{CD}-\mathrm{ROM}$ products have been proposed, they tend to cover a broad range of features of diverse nature and pay very little or no attention to interface issues. Besides, the professional literature argues that there is a general lack of attention paid to interface issues by $\mathrm{CD}$ - ROM producers and that $\mathrm{CD}$ - $\mathrm{ROM}$ product reviews published in professional journals have been uncritical in their discussion of the user interface. If we consider CDROM as an end user application, the importance of the interface is paramount. Therefore, HumanComputer Interaction (HCI) evaluation principles and methods were applied to CD-ROMs. After all, they are no different from other interactive computer systems.

\section{Methodology}

The project was undertaken in three stages: interface evaluation model, product sample, and expert evaluation.

The first stage involved the building of an evaluation model to identify the interface components to be assessed. Its aim was to create an appropriate framework to collect comparative data across the sample of user interfaces. The evaluation focused primarily on HCI practice and the concept of usability. It also drew on written guidelines and style guides, including general interface design guidelines, specific Windows applications design guidelines and The CD-ROM consistent interface guidelines.

The model identified two main levels of interaction for evaluation: the dialogue level interaction and the task level interaction. The first level includes general interface features within the GUI environment, covering elements of good HCI practice applicable to any interactive systems in general. Features evaluated included: integration of dialogue styles; form-filling templates; menu design; use of icons; use of windowing techniques; navigation and orientation. The second level focuses specifically on the HCI features which support the search and retrieval tasks and output and processing features. It also includes the system's help facilities which are intended to support the user in carrying out both the more ergonomic and conceptual tasks.

In a second stage a sample of CD-ROM products was selected. The final list comprises seven CDROM products, including full text and bibliographic databases ${ }^{(2)}$. We focused on Windows based applications since these have become more widespread and provide a standard platform and environment for interface design. 
In the third stage an 'expert evaluation' was carried out. An expert appraisal of a system involves an appraisal of system conformance, where the expert compares the system with established human factors standards, guidelines and principles, which were embodied in the evaluation model. The main strength of this method is that it is strongly diagnostic and predictive. Its aim is to attempt to predict aspects of the usage of a system prior to observing it directly in an experimental or operational setting. It is recognized that in this type of evaluation exercise, where there is only one evaluator playing the role of a user (I had extensive previous experience with CD-ROM products and users) a major limitation could be the possibility of bias or subjectivity. The results are validated not only by the systematic and comparative approach but are also intended to anticipate some of the problems users are likely to encounter when interacting with the systems. Expert evaluation does not replace the participation of users in the evaluation process. In fact, in the current study the expert evaluation is viewed as a precursor to a user study.

The applications were evaluated in turn and individual results were then gathered together to provide data for comparison. The discussion of the results focuses on design limitations and a number of recommendations for the improvement of the interface design were made.

\section{Conclusions}

The study produced recommendations for improvement of interface design and identified areas for further research (which will involve observation of users in operational settings or/and laboratorytype conditions). The evaluation's most outstanding conclusions were the lack of understanding of the Information Retrieval (IR) task -the applications follow general purpose software models and metaphors and therefore do not address the fundamental issue of information retrieval as an inherently interactive task- and the lack of attention to user needs. Because applications are still designed with the professional user in mind, they tend to include too many functionality options without an appropriate presentation of options to the user. Clearly there is an urgent need to base design decisions on empirical evidence drawn from end users.

GUIs offer much scope for designing more effective interfaces, but the cosmetic appeal of GUIs to serve as a tool to tailor the task is no substitute for the need for systems designers to tackle the real design work of tailoring the tool to the user's task.

1. Manglano Bosch, V. and Hancock-Beaulieu, M. (1995) CD-ROM User Interface Evaluation: the Appropriateness of GUIs, Online \& CD-ROM Review, Vol. 19 (5), pp. 255-270.

2. The Economist on CD-ROM (Windows Personal Librarian); Admyte (Collection of Spanish Manuscripts); The English Poetry; Oxford English Dictionary 2nd ed. on CD-ROM; Perinorm (CD-Answer for Windows), OVID for Windows Medline; WinSPIRS Medline. 